\title{
Targeted co-delivery of Beclin I siRNA and FTY720 to hepatocellular carcinoma by calcium phosphate nanoparticles for enhanced anticancer efficacy
}

This article was published in the following Dove Press journal: International Journal of Nanomedicine

\author{
Jun-Yi Wu ${ }^{1, *}$ \\ Zhong-Xia Wangl,* \\ Guang Zhang' \\ Xian Lu' \\ Guang-Hui Qiang² \\ Wei $\mathrm{Hu}^{2}$ \\ An-Lai Ji ${ }^{3}$ \\ Jun-Hua $\mathrm{Wu}^{4}$ \\ Chun-Ping Jiang'
}

'Department of Hepatobiliary Surgery, Affiliated Drum Tower Hospital of Nanjing University Medical School, Nanjing, Jiangsu, China; ${ }^{2}$ Department of Hepatobiliary Surgery, Drum Tower Clinical College of Nanjing Medical University, Nanjing, Jiangsu, China; ${ }^{3}$ Department of General Surgery, The Affiliated Hospital of Yangzhou University, Yangzhou University, Yangzhou, Jiangsu, China; ${ }^{4}$ Jiangsu Key Laboratory of Molecular Medicine, Medical School, Nanjing University, Nanjing, Jiangsu, China

*These authors contributed equally to this work
Purpose: FTY720, known as fingolimod, is a new immunosuppressive agent with effective anticancer properties. Although it was recently confirmed that FTY720 inhibits cancer cell proliferation, FTY720 can also induce protective autophagy and reduce cytotoxicity. Blocking autophagy with Beclin 1 siRNA after treatment with FTY720 promotes apoptosis. The objective of this study was to enhance the anticancer effect of FTY720 in hepatocellular carcinoma (HCC) by targeted co-delivery of FTY720 and Beclin 1 siRNA using calcium phosphate (CaP) nanoparticles (NPs).

Materials and methods: First, the siRNA was encapsulated within the CaP core. To form an asymmetric lipid bilayer structure, we then used an anionic lipid for the inner leaflet and a cationic lipid for the outer leaflet; after removing chloroform by rotary evaporation, these lipids were dispersed in a saline solution with FTY720. The NPs were analyzed by transmission electron microscopy, dynamic light scattering and ultraviolet-visible spectrophotometry. Cancer cell viability and cell death were analyzed by MTT assays, fluorescence-activated cell sorting analysis and Western blotting. In addition, the in vivo effects of the NPs were investigated using an athymic nude mouse subcutaneous transplantation tumor model.

Results: When the CaP NPs, called LCP-II NPs, were loaded with FTY720 and siRNA, they exhibited the expected size and were internalized by cells. These NPs were stable in systemic circulation. Furthermore, co-delivery of FTY720 and Beclin 1 siRNA significantly increased cytotoxicity in vitro and in vivo compared with that caused by treatment with the free drug alone.

Conclusion: The CaP NP system can be further developed for co-delivery of FTY720 and Beclin 1 siRNA to treat HCC, enhancing the anticancer efficacy of FTY720. Our findings provide a new insight into HCC treatment with co-delivered small molecules and siRNA, and these results can be readily translated into cancer clinical trials.

Keywords: LCP-II NPs, autophagy, FTY720, Beclin 1, co-delivery

\section{Introduction}

With 700,000 deaths annually, hepatocellular carcinoma (HCC) is the fifth most common malignancy in the world and a serious threat to human health. ${ }^{1}$ The development and progression of HCC are associated with multiple genetic mutations and genetic heterogeneity. ${ }^{2}$ At present, the most effective treatment for HCC is surgical resection and liver transplantation. ${ }^{3-6}$ However, surgical resection and liver transplantation still faces major problems, such as tumor recurrence and metastasis. ${ }^{4}$ In addition, surgical therapy is always ineffective for patients with advanced HCC. ${ }^{5}$ Therefore, it is necessary to develop novel therapies for HCC treatment. ${ }^{5}$ 
FTY720 (fingolimod), a Federal Drug Administrationapproved drug and the first oral therapeutic for multiple sclerosis, was recently confirmed to possess strong antitumor effects. ${ }^{7-9}$ Compared with traditional chemotherapies, FTY720 may be a beneficial anticancer drug because it is less toxic and has better oral bioavailability. Although FTY720 has been widely used in various cancers, an increasing number of studies have found that FTY720 induces cell autophagy, adversely affecting apoptosis and contributing to tumor resistance. ${ }^{10-12}$ Autophagy in tumor therapy is associated with chemotherapy, radiation and immune tolerance. ${ }^{13,14}$ In addition, increased autophagy after treatment with anticancer drugs reduces the effect of drugs. ${ }^{15,16}$ Furthermore, some studies have confirmed that blocking autophagy through Beclin 1 siRNA (siBeclin 1) or ATG 5 siRNA (siATG 5) increases cancer cell sensitivity to FTY720. ${ }^{10,17-19}$ Gene silencing strategies, particularly RNA interference, have been widely applied to inhibit the expression of cancerrelated proteins. ${ }^{20,21}$ Combining drug and siRNA therapy to downregulate cancer-related gene expression, promoting the anticancer drug effects at the tumor site can effectively suppress tumor progression, and an increasing number of studies have focused on using nonviral vectors to co-deliver drugs and siRNA. ${ }^{22-25}$ Incorporating drugs and siRNA into one vector is the most obvious method for achieving a true synergistic effect from simultaneous delivery of both a drug and an siRNA. ${ }^{26}$ The objective of this study was to co-deliver FTY720 and siRNA to enhance the effect of this anticancer drug.

Constructing a co-delivery vector has several challenges, which are as follows: the drug and siRNA must be loaded at a high entrapment rate, the carrier needs to remain stable in systemic circulation and targeted release of the drug and siRNA at the tumor site must be achieved. ${ }^{27}$ In recent studies, nanoparticles (NPs), which are nanoscale carriers, have demonstrated great value and potential for promoting drug/gene delivery. ${ }^{28-30}$ For instance, Kang et al confirmed that cationic liposomal co-delivery of siRNA and an MEK inhibitor improved anticancer efficacy. ${ }^{31}$ In addition, Ding et al designed a nanostructure-based drug and siRNA delivery system to knock down the expression of Rap2b, a novel p53 target, enhancing adriamycin-based anticancer therapeutics. ${ }^{32}$ Furthermore, the use of a novel liposome to efficiently deliver siRNA against MGMT, a DNA repair protein, promoted temozolomide cytotoxicity, ${ }^{33}$ and the strategy of simultaneously co-delivering epirubicin and BCL-2 siRNA with lipid-NPs significantly reversed tumor cell multidrug resistance.$^{34}$ In summary, these combined therapies provide an experimental basis for their use to reduce clinical drug resistance.

Recently, Li et al designed calcium phosphate (CaP) NPs with an asymmetric lipid bilayer coating for targeted delivery of siRNA to tumors..$^{35}$ An anionic lipid, dioleoylphosphatidic acid (DOPA), was used as the inner leaflet lipid to coat the nanosized $\mathrm{CaP}$ cores, thereby entrapping the siRNA and making the coated cores soluble in organic solvent; a suitable neutral or cationic lipid was used as the outer leaflet lipid to form an asymmetric lipid bilayer structure. The formulation was named lipid/calcium/phosphate type II (LCP-II). The results showed that the improved formulation effectively delivered siRNA in vitro and to the tumor site in vivo. In this study, we used LCP-II with minor modifications to co-deliver Beclin 1 siRNA and FTY720 (termed LCP-IIsiBeclin 1-FTY720) to enhance cytotoxicity by blocking the protective autophagy induced by FTY720. LCP-II has great potential for simultaneously transporting siRNA and FTY720 across the cell membrane to reduce Beclin 1 expression and to induce more FTY720-mediated cytotoxicity than free FTY720 alone.

\section{Materials and methods Materials}

DOPA, 1,2-dioleoyl-3-trimethylammonium propane (DOTAP), 1,2-distearoyl-sn-glycero-3-phosphoethanolamine- $N$-methoxy(polyethylene glycol-2000) ammonium salt (DSPE-PEG) and DSPE-PEG-folic acid (DSPE-PEG-FA) were purchased from Avanti Polar Lipids, Inc. (Alabaster, AL, USA). Cholesterol was purchased from Sigma-Aldrich. Beclin 1 siRNA (target sequence 5'-CAGTTTGGCACAATC AATA-3') labeled with or without fluorescein (FAM or Cy5) and control siRNA (target sequence 5'-AATTCTCCGAACGT GTCACGT-3') labeled with or without FAM were purchased from Shanghai GenePharma Co., Ltd. (Shanghai, China).

\section{Cell lines and cell culture}

The cell lines SMMC-7721 and A549 were purchased from the Cell Bank of the Chinese Academy of Sciences (Shanghai, China) and were cultured in complete DMEM (Wisent Inc., St-Bruno, QC, Canada) supplemented with $10 \%$ fetal bovine serum (Wisent Inc.) at $37^{\circ} \mathrm{C}$ in a humidified incubator with $5 \% \mathrm{CO}_{2}$.

\section{Preparation and characterization of LCP-II NPs}

LCP-II NPs were prepared according to Huang's protocol with minor modifications as previously described. ${ }^{35}$ Figure 1 


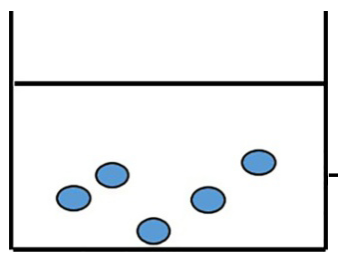

$\mathrm{CaCl}_{2}+$ siRNA



$\mathrm{HPO}_{4}^{2-}(\mathrm{pH} 9.0)+$ DOPA

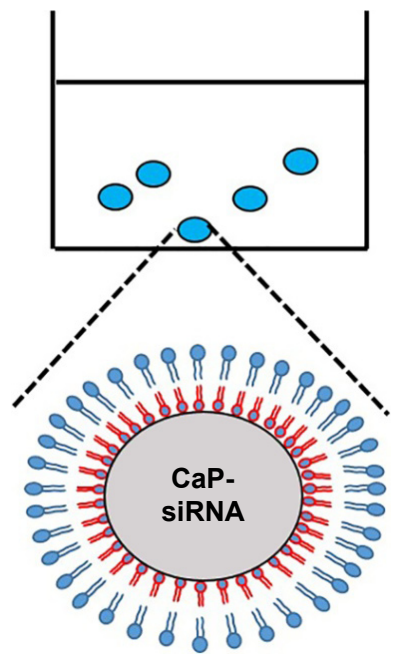

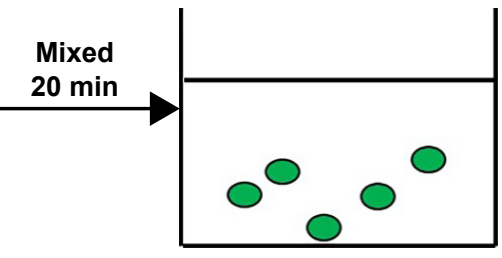

CaP core with siRNA

$12,000 \times g 20 \mathrm{~min}$

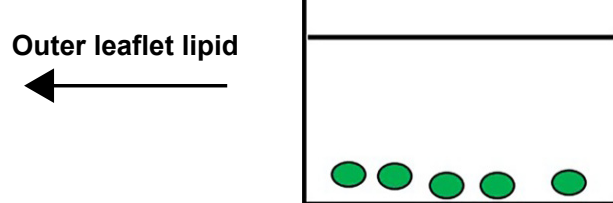

Washed with ethanol two to three times


Residual lipid was dispersed in saline with FTY720

Figure I Schematic for the preparation of LCP-II NPs for co-delivery of siRNA and FTY720.

Abbreviations: DOPA, dioleoylphosphatidic acid; FTY720, fingolimod; LCP-II NPs, lipid/calcium/phosphate type II nanoparticles.

illustrates a flow diagram of the preparation of LCP-II-siBeclin 1-FTY720. First, $300 \mu \mathrm{L}$ of $\mathrm{CaCl}_{2}(500 \mathrm{mM})$ and $100 \mu \mathrm{L}$ of siBeclin $1(2 \mathrm{mg} / \mathrm{mL})$ were dispersed in $15 \mathrm{~mL}$ of a cyclohexane/Igepal CO-520 (71/29 volume/volume [V/V]) solution to prepare the water-in-oil microemulsion. To form the phosphate portion, $300 \mu \mathrm{L}$ of $\mathrm{Na}_{2} \mathrm{HPO}_{4}(25 \mathrm{mM}, \mathrm{pH}=9.0)$ solution was added to another $15 \mathrm{~mL}$ of cyclohexane/Igepal CO-520 $(71 / 29 \mathrm{~V} / \mathrm{V})$ solution, and then, $200 \mu \mathrm{L}$ of DOPA $(20 \mathrm{mg} / \mathrm{mL})$ in chloroform was added to the phosphate phase. After the two solutions were mixed for $20 \mathrm{~min}, 30 \mathrm{~mL}$ of absolute ethanol was added to the mixture, and the resulting mixture was centrifuged at $12,000 \times \mathrm{g}$ for $20 \mathrm{~min}$ to remove the cyclohexane/ Igepal and to pellet the $\mathrm{CaP}$ core. The pellets were washed with ethanol two to three times, dissolved in $1 \mathrm{~mL}$ of chloroform and then stored in a glass vial for the upcoming procedures.
To prepare LCP-II-siBeclin 1-FTY720, $1 \mathrm{~mL}$ of CaP core, $100 \mu \mathrm{L}$ of DOTAP/cholesterol $(1: 1,10 \mathrm{mM})$ and $100 \mu \mathrm{L}$ of DSPE-PEG-2000 or DSPE-PEG-2000-FA (3 mM) were mixed together, and then, the chloroform was removed by rotary evaporation. The residual lipid was dispersed in $800 \mu \mathrm{L}$ of saline solution with $10 \mathrm{mM} \mathrm{FTY720} \mathrm{to} \mathrm{form}$ LCP-II-siBeclin 1-FTY720. The LCP-II-siBeclin 1-FTY720 NPs were extruded through 200 and $100 \mathrm{~nm}$ polycarbonate membranes three times and were dialyzed against HEPESbuffered saline (HBS, $145 \mathrm{mM} \mathrm{NaCl}$ and $20 \mathrm{mM}$ HEPES [pH 7.4]) with a molecular weight cut-off of $10 \mathrm{kDa}$ to remove non-encapsulated FTY720. LCP-II-siRNA with or without FA (DSPE-PEG-2000-FA or DSPE-PEG-2000), LCP-IIsiBeclin 1 with or without FA, LCP-II-siRNA-FTY720 with or without FA, and LCP-II-siBeclin 1-FTY720 with or 
without FA were prepared by the method mentioned above. LCP-II-siRNA (LCP-II-entrapped siRNA NPs with FA) was used as a negative control. LCP-II-siBeclin 1 represents LCP-II-entrapped siBeclin 1 NPs with FA. LCP-II-siRNAFTY720 represents LCP-II-entrapped siRNA and FTY720 NPs with FA, which was used as a negative control. LCP-IIsiBeclin 1-FTY720 represents LCP-II-entrapped siBeclin 1 and FTY720 NPs with FA. Except where noted, the different formulations were all prepared with the FA ligand.

To measure the encapsulation efficiency (EE) of FTY720 in the LCP-II NPs after they were dispersed in saline solution with $10 \mathrm{mM}$ FTY720, the LCP-II NP-containing solution was centrifuged at $12,000 \times g$ for $20 \mathrm{~min}$ and the supernatant was harvested for absorbance detection with an ultravioletvisible spectrophotometer (V500; Jasco, Tokyo, Japan). ${ }^{36}$ FTY720 in saline solution had a maximum absorption wavelength of $220 \mathrm{~nm}$. The absorbance of different samples was obtained at $220 \mathrm{~nm}$. The concentration of FTY720 was measured according to the Beer-Lambert law, and the EE was determined according to the following equation: $\mathrm{EE}=(1-$ concentration of free FTY720/concentration of total FTY720) $\times 100 \%$.

LCP-II NPs were examined by transmission electron microscopy (TEM) as previously described with small modifications. ${ }^{35}$ LCP-II NPs $(1 \mu \mathrm{L})$ were resuspended in $1 \mathrm{~mL}$ of PBS and applied to copper-mesh formvar grids (Beijing Zhongxingkeyi Technology Co., Ltd., Beijing, China) with or without negative staining with phosphotungstic acid. TEM images were obtained with a JEM-2100 transmission electron microscope (Jeol, Tokyo, Japan). For size and zeta potential measurements, $1 \mu \mathrm{L}$ of LCP-II NPs was resuspended in $1 \mathrm{~mL}$ of PBS, and this LCP-II NP-containing PBS solution was used for size and zeta potential measurements with a Zetasizer Nano ZS90 instrument (Malvern Instruments, Malvern, UK).

\section{In vitro release of FTY720 from LCP-II NPs}

To measure the release rate of FTY720 from LCP-II-siRNAFTY720 and LCP-II-siBeclin 1-FTY720, the NPs were added to $\mathrm{PBS}(\mathrm{pH}=7.4)$ at $37^{\circ} \mathrm{C}$. The unreleased FTY 720 , which was retained within the LCPII-siRNA-FTY720 and LCP-II-siBeclin 1-FTY720 NPs, was removed at different time points $(0,6,12,24,36$ and $48 \mathrm{~h})$ by centrifugation at $12,000 \times g$ for $20 \mathrm{~min}$, and then, the supernatant (the released portion) was harvested for absorbance detection by a ultraviolet-visible spectrophotometer. ${ }^{36}$ The concentration of FTY720 was measured as described above. The percentage of released FTY720 was calculated by comparing the released portion to the original total FTY720 amount. To measure the stability of LCP-II NPs in culture medium, the NPs were added to the culture medium for 6 and $12 \mathrm{~h}$ and the sizes of LCP-II-siBeclin 1 and LCP-II-siBeclin 1-FTY720 were measured with a Zetasizer Nano ZS90 instrument (Malvern Instruments).

\section{Cell uptake of LCP-II NPs}

SMMC-7721 cells $\left(1 \times 10^{5}\right.$ per well $)$ were cultured on coverslips in 12-well plates. After $24 \mathrm{~h}$, the cells were treated with LCP-II-siRNA with or without FA, LCP-II-siBeclin 1 with or without FA or LCP-II-siBeclin 1-FTY720 with or without $\mathrm{FA}$ at $37^{\circ} \mathrm{C}$ for $4 \mathrm{~h}(100 \mathrm{nM}$ total siRNA labeled with FAM) and then washed with PBS twice. The cells were further fixed with $4 \%$ paraformaldehyde at room temperature for $20 \mathrm{~min}$ and, again, were washed with PBS twice. After being counterstained with DAPI, cell images were obtained using a confocal microscope (FV10i; Olympus Corporation, Tokyo, Japan).

\section{Cell viability analysis}

To measure the cell viability in vitro, SMMC-7721 cells were cultured in 96-well plates at a concentration of $1 \times 10^{4}$ cells/well. After $24 \mathrm{~h}$, the cells were treated with PBS, free FTY720 solution, solution containing free FTY720 and lipo2000-siBeclin 1, LCP-II-siRNA, LCP-II-siBeclin 1, LCP-II-siRNA-FTY720, LCP-II-siBeclin 1-FTY720 without FA or LCP-II-FTY720-siBeclin 1 with FA for $24 \mathrm{~h}$ (100 nM total siRNA). Lipo2000-siBeclin 1 represents the cells transfected with siBeclin 1 using Lipofectamine 2000 (Thermo Fisher Scientific, Waltham, MA, USA) in accordance with the manufacturer's instructions. Then, MTT solution ( $5 \mathrm{mg} / \mathrm{mL}, 20 \mu \mathrm{L} /$ well) was added to the 96-well plates. After incubation for $4 \mathrm{~h}, 150 \mu \mathrm{L}$ of dimethyl sulfoxide was added to dissolve the insoluble crystals. Absorbance values were measured with a spectrophotometer at $490 \mathrm{~nm}$. All the experiments were performed with three replicates.

\section{Flow cytometric analysis}

SMMC-7721 cells $\left(1 \times 10^{6}\right)$ were seeded in six-well plates for $24 \mathrm{~h}$ and treated with PBS control, LCP-II-siBeclin 1, LCPII-siBeclin 1-FTY720 with or without FA or LCP-II-siBeclin 1-FTY720 plus $1 \mathrm{mM} \mathrm{FA}$ at $37^{\circ} \mathrm{C}$ for $4 \mathrm{~h}(100 \mathrm{nM}$ total siRNA labeled with FAM). A549 cells $\left(1 \times 10^{6}\right)$ were seeded in six-well plates for $24 \mathrm{~h}$ and treated with PBS control or LCP-II-siBeclin 1-FTY720 with FA at $37^{\circ} \mathrm{C}$ for $4 \mathrm{~h}(100 \mathrm{nM}$ total siRNA labeled with FAM). The uptake of LCP-II NPs 
by SMMC-7721 and A549 cells was confirmed qualitatively by flow cytometry (FACSCalibur; BD, Franklin Lakes, NJ, USA). To evaluate the apoptosis rate, SMMC-7721 cells $\left(1 \times 10^{6}\right)$ were seeded in six-well plates for $24 \mathrm{~h}$ and treated with a solution of free FTY720, a solution containing both free FTY720 and lipo2000-siBeclin 1, or one of the following NP solutions for $24 \mathrm{~h}$ : LCP-II-siRNA, LCP-II-siBeclin 1, LCP-II-siRNA-FTY720, LCP-II-siBeclin 1-FTY720 without FA or LCP-II-FTY720-siBeclin 1 with FA (100 nM total siRNA, 7.5 $\mu \mathrm{M}$ FTY720). Cells, including apoptotic, dead and adherent cells, were collected and washed with cold PBS and then resuspended in annexin V-fluorescein isothiocyanate (FITC) binding buffer. After incubation with annexin V-FITC/propidium iodide (PI), apoptosis was detected by flow cytometry.

\section{Western blot analysis}

After being treated with free FTY720 solution, solution containing both free FTY720 and lipo2000-siBeclin 1, or LCP-II-siRNA, LCP-II-siBeclin 1, LCP-II-siRNA-FTY720, LCP-II-siBeclin 1-FTY720 without FA or LCP-II-FTY720siBeclin 1 with FA NPs for $24 \mathrm{~h}(100 \mathrm{nM}$ total siRNA, $7.5 \mu \mathrm{M}$ FTY720), the SMMC-7721 cells were lysed with radioimmunoprecipitation assay peptide lysis buffer (Beyotime Biotechnology, Haimen, China) containing 1\% protease inhibitors (Thermo Fisher Scientific). After the proteins were quantified through a BCA protein assay kit (Thermo Scientific Fisher Scientific), they were fractionated by sodium dodecyl sulfatepolyacrylamide gel electrophoresis. Then, the proteins were stained with antibodies targeting Beclin 1, LC3B, PARP, cleaved PARP, caspase-9, cleaved caspase-9 and cleaved caspase-3 from Cell Signaling Technology (Beverly, MA, USA); the dilution of the antibodies used was 1:1,000. The control antibody (anti-GAPDH, 1:10,000) was from Bioworld Technology, Inc. (St. Louis Park, MN, USA). The protein bands were detected using enhanced chemiluminescence (EMD Millipore, Billerica, MA, USA) after incubation with horseradish peroxidase-conjugated secondary antibodies.

\section{Quantitative real-time-polymerase chain reaction analysis}

SMMC-7721 cells were seeded and transfected with lipo2000-siBeclin 1, LCP-II-siRNA, LCP-II-siBeclin 1, LCP-II-siBeclin 1-FTY720 without FA or LCP-II-FTY720siBeclin 1 with FA NPs for $24 \mathrm{~h}(100 \mathrm{nM}$ total siRNA, $7.5 \mu \mathrm{M}$ FTY720). RNA was extracted, purified and converted to cDNA with an RNAiso Plus kit and PrimeScript RT Master Mix (Takara Bio Inc., Naha, Japan). Quantitative polymerase chain reaction was performed using SYBR Premix Ex Taq II (Takara Bio Inc.).

\section{Tumor accumulation studies}

All animal studies were conducted according to the national guidelines of the Institutional Animal Care and Use Committee and were approved by the Animal Care Ethics Committee of Nanjing Drum Tower Hospital. Four- to six-week-old male nude mice (BALB/c nude mice) were purchased from the Laboratory Animal Center of Shanghai, Academy of Science. To evaluate tumor-targeting ability, SMMC-7721 cells were resuspended in PBS and injected into the right axillary region of mice after infiltration anesthesia with isoflurane ( $1 \times 10^{7}$ cells in $200 \mu \mathrm{L}$ of PBS per mouse). When the tumor grew to $\sim 800 \mathrm{~mm}^{3}$, the mice were injected intravenously with PBS, LCP-II-siBeclin 1 (Cy5 labeled), LCP-II-siBeclin 1FTY720 (Cy5 labeled) or LCP-II-siBeclin 1-FTY720 (Cy5 labeled) without FA at the given FTY720 (5 mg/kg) and siRNA ( $2 \mathrm{mg} / \mathrm{kg}$ ) dose. After $12 \mathrm{~h}$, the Cy5 fluorescence signals throughout the whole body were observed with an IVIS Lumina III system (PerkinElmer, Akron, OH, USA).

\section{Mice and in vivo tumor studies}

SMMC-7721 cells were resuspended in PBS and injected in the right axillary region of mice $\left(1 \times 10^{7}\right.$ cells in $200 \mu \mathrm{L}$ of PBS per mouse). After 2 weeks, when the tumor volumes were $\sim 100 \mathrm{~mm}^{3}$, the mice were randomly divided into five groups (25 mice in total, with five in each of the following groups: PBS, LCP-II negative control, LCP-II-siRNAFTY720, LCP-II-siBeclin 1-FTY720 without FA and LCP-II-siBeclin 1-FTY720 with FA) and were injected daily through the tail vein with different complexes $(5 \mathrm{mg} / \mathrm{kg}$ total FTY720, $2 \mathrm{mg} / \mathrm{kg}$ siBeclin 1) for 3 weeks. The longest (a) and the shortest (b) tumor diameters were examined every 4 days with a Vernier caliper. The tumor volume $\left(\mathrm{mm}^{3}\right)=1 / 2 \times a \times b \times b$, and all the mice were sacrificed 35 days after the subcutaneous SMMC-7721 cell injection.

\section{Statistical analysis}

All results are expressed as the mean \pm SD. The data were analyzed with Student's $t$-test. When $P<0.05$, the data were considered significantly different.

\section{Results \\ Preparation and characterization of LCP-II NPs}

LCP-II NPs were prepared as previously described, with minor modifications. ${ }^{35}$ Briefly, we prepared a $\mathrm{pH}$-sensitive $\mathrm{CaP}$ core encapsulating siRNA and added the amphiphilic 
phospholipid DOPA as a precoating reagent. The CaP core was mixed with DOTAP/cholesterol (1:1) and DSPE-PEG2000 with or without FA to synthesize the LCP-II NPs. After removing chloroform with rotary evaporation, the remaining lipid was dispersed in saline with or without FTY720 to form the LCP-II-siBeclin 1-FTY720 co-delivery NPs (Figure 1). Finally, the NPs were extruded through 200 and $100 \mathrm{~nm}$ polycarbonate membranes three times. The size, morphology and surface charge of the LCP-II NPs were determined by TEM (Figure 2A) and a Zetasizer Nano ZS90 instrument. After three extrusion cycles through the 200 and $100 \mathrm{~nm}$ polycarbonate membranes, the average particle size of the LCP-II-siRNA NPs was $57 \pm 6.4 \mathrm{~nm}$ (Figure 2B), and after loading with FTY720, the size was $76 \pm 3.9 \mathrm{~nm}$ (Figure 2B). The zeta potential was $\sim 5 \mathrm{mV}$ for LCP-II-siRNA NPs and $12 \mathrm{mV}$ for LCP-II-siRNA-FTY720
NPs (Figure 2C), suggesting that FTY720 loading slightly influenced the surface zeta potential of the complexes. The EE of LCP-II NPs loaded with FTY720 was 36.1\% $2.4 \%$. To determine the in vitro release kinetics of FTY720 from the LCP-II-siRNA-FTY720 NPs, the formulation was incubated in PBS $(\mathrm{pH}=7.4)$ at $37^{\circ} \mathrm{C}$. The FTY720 released from the LCP-II-siRNA-FTY720 NPs into the medium after $12 \mathrm{~h}$ was $<30 \%$, but after $24 \mathrm{~h}$, the LCP-II NPs still retained over $45 \%$ of the FTY720 (Figure 2D). In addition, the amount of FTY720 released from LCP-II-siBeclin 1-FTY720 NPs was similar to the amount released from LCP-II-siRNAFTY720 NPs (Figure 2D). Furthermore, the sizes of LCP-IIsiBeclin 1 and LCP-II-siBeclin 1-FTY720 remained stable in culture medium (Figure 2E). These results indicated that the LCP-II-siRNA-FTY720 and LCP-II-siBeclin 1-FTY720 NPs were stable in PBS and culture medium.
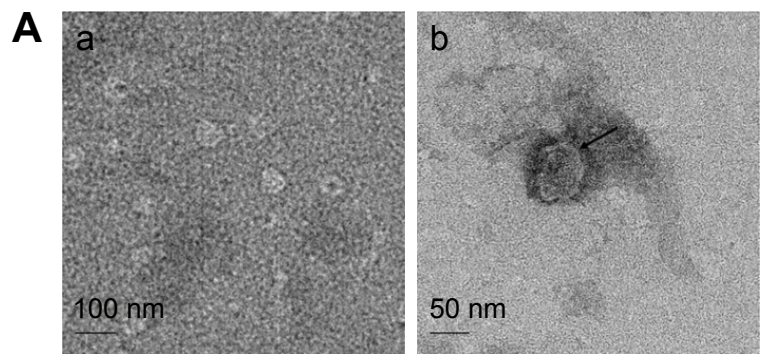

B

LCP-II-siRNA
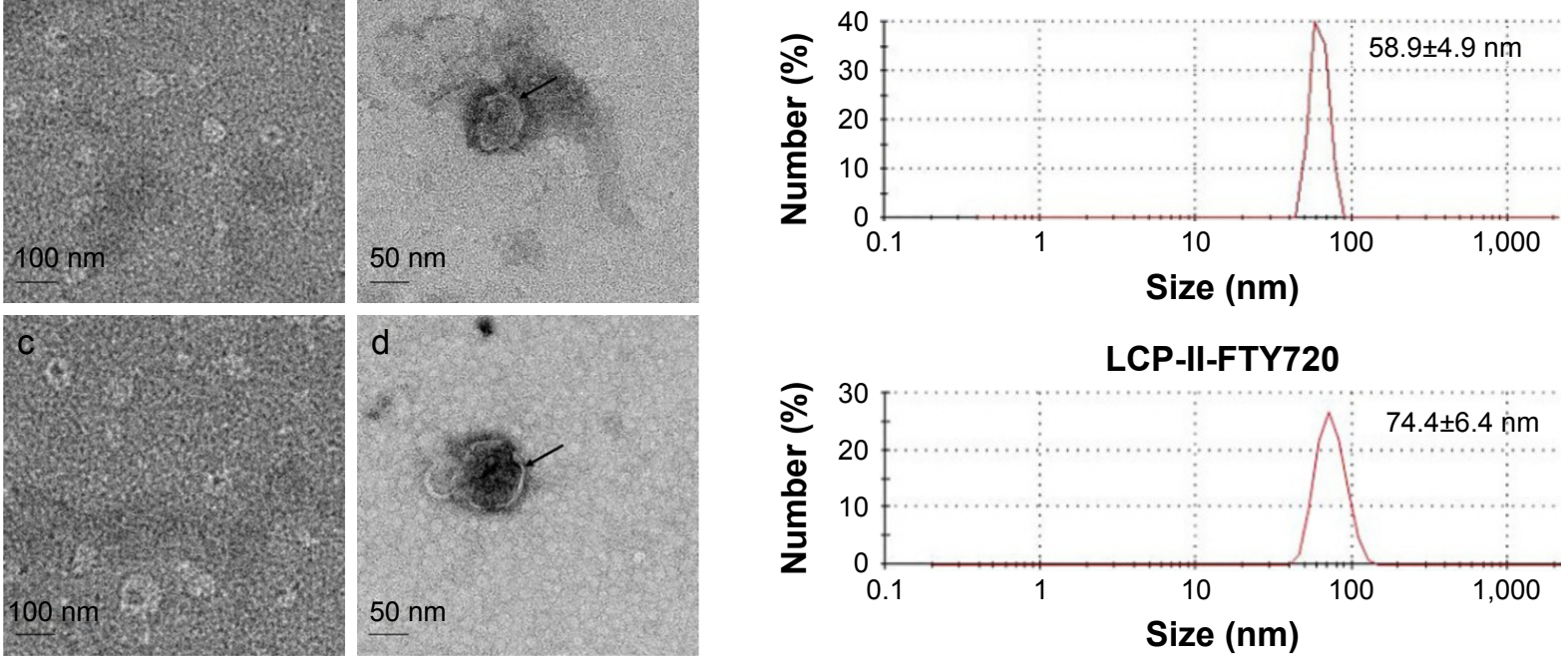

C Zeta potential

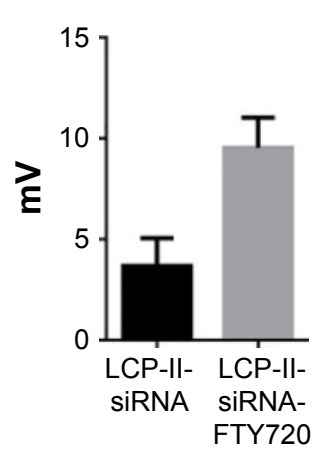



E

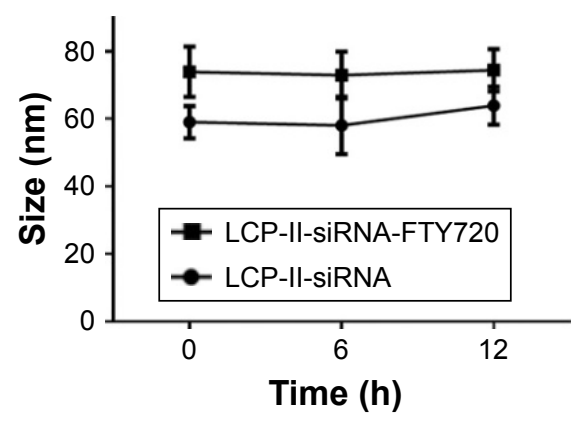

Figure 2 Characterization of LCP-II NPs loaded with siRNA and FTY720.

Notes: (A) Representative TEM images of LCP-II-siRNA (a) without and (b) with negative staining and LCP-II-siRNA-FTY720 NPs (c) without and (d) with negative staining. Arrows in (b, d) show the lipid bilayer surrounding the CaP core. (B, C) Size and zeta potential distribution of LCP-II-siRNA and LCP-II-siRNA-FTY720. (D) FTY720 released from LCP-II-siRNA-FTY720 and LCP-II-siBeclin I-FTY720 NPs over time in vitro. (E) The sizes of LCP-II-siBeclin I and LCP-II-siBeclin I-FTY720 in the culture medium.

Abbreviations: FTY720, fingolimod; LCP-II NPs, lipid/calcium/phosphate type II nanoparticles; siBeclin I, Beclin I siRNA; TEM, transmission electron microscopy. 


\section{Cellular uptake of LCP-II NPs and the in vitro gene silencing activity of LCP-II NP-delivered siBeclin I}

The folate receptor (FR) is highly expressed in various cancers, including HCC, and has low expression levels in normal tissue.${ }^{37} \mathrm{FA}$, a necessary vitamin for human cells, has a high affinity for the FR. In addition, coupling FA to other molecules maintains the affinity of FA for FR, and FA can be internalized through cell endocytosis. To achieve ligandmeditated siRNA and drug uptake in target cells, FA was coupled to the distal end of DSPE-PEG, which is compatible with FR as a targeting ligand. The cellular uptake of LCP-II NPs by SMMC-7721 cells was evaluated using fluorescence microscopy. siRNA and siBeclin 1 were labeled with FAM. LCP-II-siRNA NPs with or without FA, LCP-II-siBeclin 1 NPs with or without FA or LCP-II-siBeclin 1-FTY720 NPs with or without FA were added to SMMC-7721 cells. After $4 \mathrm{~h}$, images of the SMMC-7721 cells were collected. The results demonstrated that SMMC-7721 cells presented with green intracellular fluorescence following incubation with FAM-siRNA-loaded LCP-II NPs with FA, indicating that FAM-labeled siRNA was evenly distributed throughout the cytoplasm of SMMC-7721 cells (Figure 3A). However, when the LCP-II NPs that were not tethered to FA were used, they were not easily internalized by the cells (Figure 3B). In addition, LCP-II-siBeclin 1 and LCP-II-siBeclin 1-FTY720 were effectively internalized by the cells, indicating that co-loading siRNA and FTY720 into the LCP-II NPs did not affect NP function (Figure 3C-F). In addition, the uptake of LCP-IIsiBeclin 1-FTY720 by SMMC-7721 cells was confirmed qualitatively by flow cytometry (Figure $3 \mathrm{G}$ ). The results showed that the co-delivery nanocarrier was effectively taken up by SMMC-7721 cells, but not when LCP-II was not tethered with FA. Moreover, to confirm that FA is a targeting ligand that improves cellular uptake, $1 \mathrm{mM}$ free FA was added to the cells, and the results showed that the FR targeting effect was blocked by $1 \mathrm{mM}$ free FA (Figure 3G). Furthermore, we used A549 cells, which are FR-negative tumor cells, ${ }^{38,39}$ to measure the cellular uptake of LCP-II NPs. The results showed that the uptake of LCP-II-siBeclin 1-FTY720 by A549 cells was very low (Figure $3 \mathrm{H}$ ), which confirmed that the FR-targeted nanocarriers showed delivery specificity to FR-positive cells, but not FR-negative cells. Moreover, the expression of Beclin 1 was significantly inhibited after treatment with LCP-II-siBeclin 1 and LCP-II-siBeclin 1-FTY720, as measured by Western blotting (Figure 3I). In addition, the Beclin 1 mRNA level was inhibited (Figure 3J). Most interestingly, the effect of LCP-II-siBeclin 1 and LCP-II-siBeclin
1-FTY720 on Beclin 1 expression was greater than that of lipo2000-siBeclin 1. Furthermore, the gene silencing activity of siBeclin 1 was greatly reduced when we used LCP-IIsiBeclin 1-FTY720 without FA, instead of LCP-II-siBeclin 1-FTY720. In conclusion, the results confirmed that LCP-II NPs can be internalized by cells.

\section{In vitro cytotoxicity of LCP-II NPs}

After treatment with the PBS control, LCP-II-siRNA or LCP-II-siBeclin $1 \mathrm{NPs}$ for $24 \mathrm{~h}$, cell viability was determined by MTT assays. The results indicated that LCP-II NPs without FTY720 did not affect cell proliferation (Figure 4A). To evaluate the cytotoxicity of co-delivered siBeclin 1 and FTY720 with LCP-II NPs compared with that of the other formulations, SMMC-7721 cells were treated with different formulations at different concentrations for $24 \mathrm{~h}$, and the inhibitory effect on cell growth was determined by MTT assays. The results showed that compared with free FTY720 or the different formulations used to treat SMMC-7721 cells, the co-delivery NPs induced the lowest cell viability. Cell growth was inhibited by $33 \%$ with $7.5 \mu \mathrm{M}$ free FTY720. When Beclin 1 expression, which is a mediator of cell autophagy, was blocked with lipo2000-siBeclin 1, the autophagy induced by FTY720 was significantly inhibited and cell cytotoxicity was further increased. The cell growth inhibition increased to $\sim 4 \%$ after protective autophagy was impaired. After treatment with LCP-II-entrapped FTY720 NPs, cell growth was inhibited by $\sim 50 \%$. More importantly, cell growth was inhibited by $65 \%$ after treatment with LCP-IIsiBeclin 1-FTY720 NPs, which effectively co-delivered siBeclin 1 and drug to the target cells. However, without the FA ligand, the cytotoxicity of the co-delivery LCP-II NPs decreased significantly. In summary, the results showed that co-delivery of FTY720 and siBeclin 1 with LCP-II NPs induced the highest level of inhibition and the highest levels of cytotoxicity (Figure 4B).

\section{GFP-LC3-transfected SMMC-772I cells treated with LCP-II NPs}

To investigate whether LCP-II-siBeclin 1-FTY720 NPs effectively blocked the cell autophagy induced by FTY720, SMMC-7721 cells were transfected with GFP-LC3, which is a well-known indicator of cell autophagy. Endogenous LC3 that is diffusely distributed in the cytoplasm organizes into punctate structures, with the occurrence of autophagy. The results showed that after treatment with free FTY720 and LCP-II-siRNA-FTY720 NPs, bright green fluorescent dots were present in SMMC-7721 cells, confirming that FTY720 


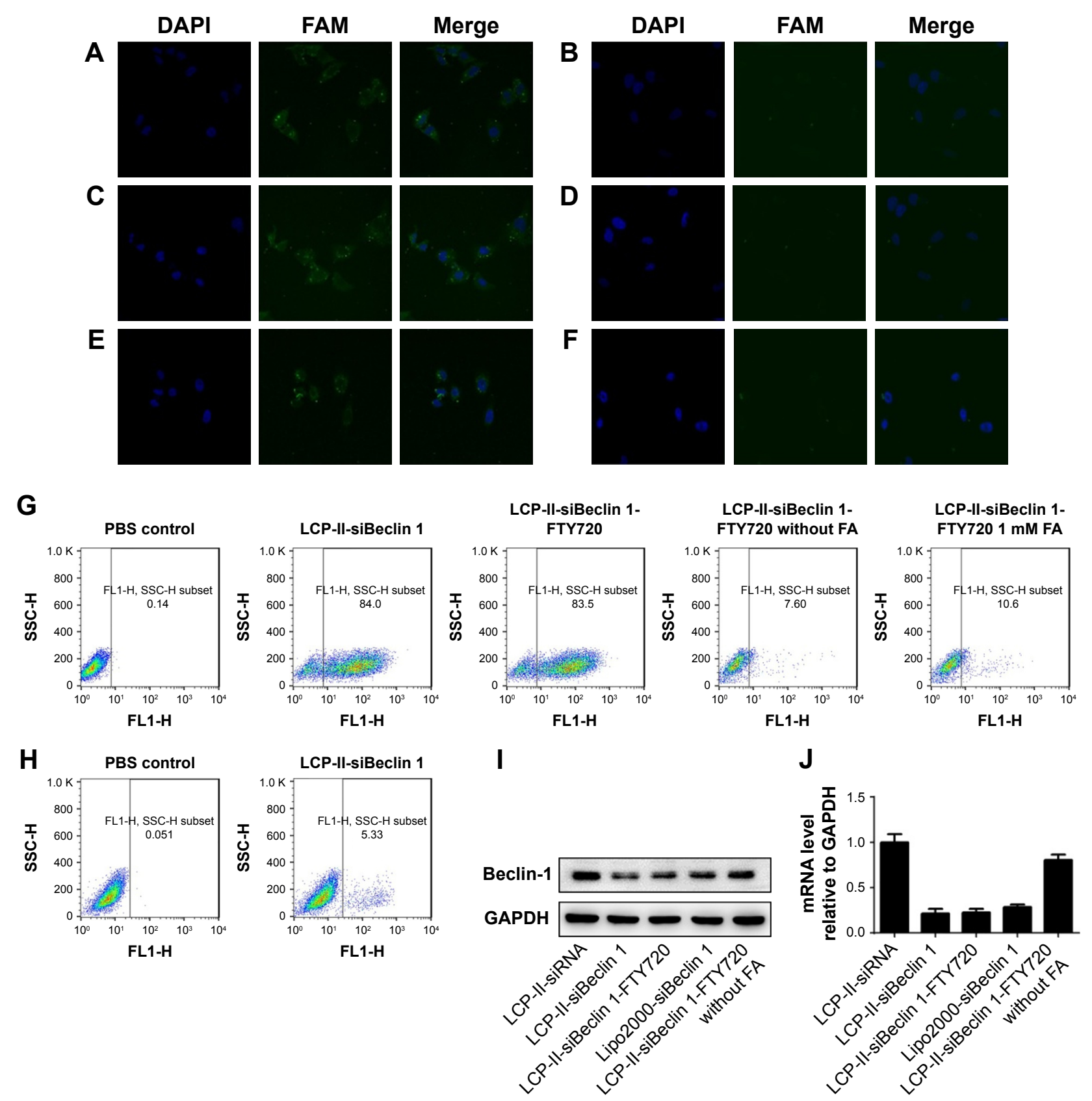

Figure 3 Cellular uptake and distribution of FAM-labeled siRNA encapsulated within LCP-II NPs.

Notes: (A) Image of LCP-II-siRNA NPs with FA targeting. (B) Image of LCP-II-siRNA NPs without FA targeting. (C) Image of LCP-II-siBeclin I NPs with FA targeting. (D) Image of LCP-II-siBeclin I NPs without FA targeting. (E) Image of LCP-II-siBeclin I-FTY720 NPs with FA targeting. (F) Image of LCP-II-siBeclin I-FTY720 NPs without FA targeting. (G) The uptake of LCP-II NPs by SMMC-772I cells was qualitatively confirmed by flow cytometry. (H) The uptake of LCP-II NPs by A549 cells was qualitatively confirmed by flow cytometry. (I) Western blot of Beclin I expression in SMMC-772I cells after treatment with LCP-II-siRNA, LCP-II-siBeclin I, LCP-II-siBeclin I-FTY720, lipo2000-siBeclin I or LCP-II-siBeclin I-FTY720 NPs without FA. (J) The Beclin I mRNA level in SMMC-772I cells after treatment with LCP-II-siRNA, LCP-II-siBeclin I, LCP-II-siBeclin I-FTY720, lipo2000-siBeclin I or LCP-II-siBeclin I-FTY720 NPs without FA.

Abbreviations: FA, folic acid; FTY720, fingolimod; LCP-II NPs, lipid/calcium/phosphate type II nanoparticles; lipo2000, Lipofectamine 2000; siBeclin I, Beclin I siRNA.

induced cell autophagy (Figure 5A). After treatment with LCP-II-FTY720 NPs for $24 \mathrm{~h}$, the proportion of SMMC-7721 cells forming LC3 puncta was $63.3 \%$, which was higher than the percentage of apoptotic cells resulting from treatment with free FTY720. Compared with LCP-II-siRNA-FTY720 NPs, treatment with LCP-II-siBeclin 1-FTY720 observably reduced the number of green fluorescent aggregates of endogenous LC3 in SMMC-7721 cells, which confirmed that LCP-II-siBeclin 1-FTY720 NPs blocked FTY720-induced autophagy. In addition, when LCP-II-siBeclin 1-FTY720 NPs without FA were added to the cells, the proportion of SMMC-7721 cells with typical LC3 puncta was reduced 
A

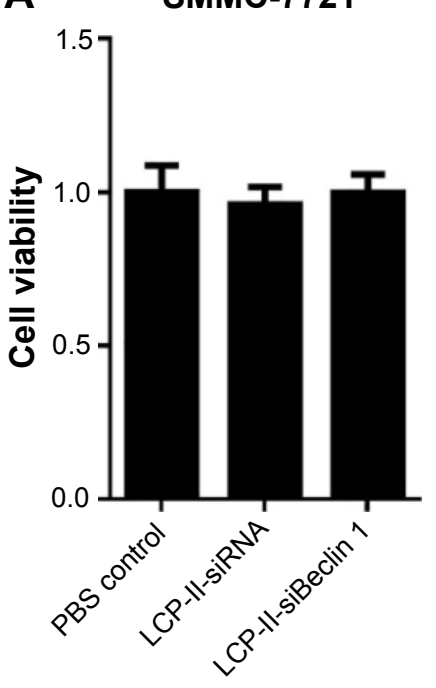

B

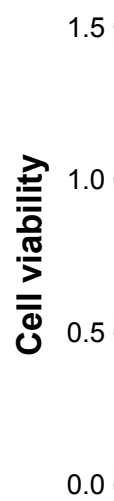

SMMC-7721
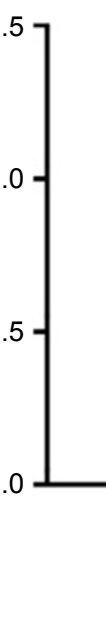

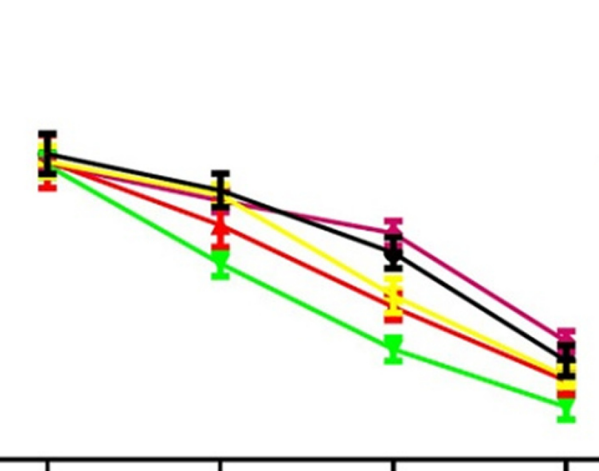

๑ Free FTY720

FTY720 + lipo2000siBeclin 1

๘ LCP-II-FTY720

- LCP-II-FTY720-siBeclin 1 with FA

$\rightarrow$ LCP-II-FTY720-siBeclin 1 without FA

Figure 4 Cell viability after treatment with different formulations.

Notes: (A) After treatment with PBS control, LCP-II-siRNA or LCP-II-siBeclin I NPs for 24 h, cell viability was determined. (B) After treatment with various FTY720 formulations for $24 \mathrm{~h}$, the cell viability of SMMC-772I cells was determined.

Abbreviations: FA, folic acid; FTY720, fingolimod; LCP-II NPs, lipid/calcium/phosphate type II nanoparticles; lipo2000, Lipofectamine 2000; siBeclin I, Beclin I siRNA.

to $34.6 \%$, which indicated that without FA targeting, the co-delivered LCP-II NPs were not internalized by the cells (Figure 5B). In addition, in the presence of autophagy, LC3-I is converted to LC3-II, an autophagy indicator. The level of LC3-II was determined by Western blotting, and the results showed that after treatment with free FTY720 and LCP-II-siRNA-FTY720 NPs, much of the LC3-I had been converted to LC3-II. When cells were treated with LCP-IIsiBeclin 1-FTY720 NPs, the levels of LC3-II and Beclin 1 were significantly decreased (Figure 5C). In summary, codelivery of siBeclin 1 and FTY720 effectively blocked the cell autophagy induced by FTY720.

\section{Apoptosis assay}

To evaluate the synergistic effects of LCP-II-siBeclin 1FTY720 co-delivery NPs on SMMC-7721 cell apoptosis, we assessed apoptosis in SMMC-7721 cells after treatment with different NP formulations. Annexin V-FITC/PI apoptosis staining was performed, and the percentage of apoptotic cells was analyzed by flow cytometry. The apoptotic rate of SMMC-7721 cells after treatment with free FTY720 was $\sim 22 \%$. After treatment with LCP-II-siRNA-FTY720 NPs, the percentage of apoptotic cells increased to $36 \%$. When Beclin 1 protein was suppressed in SMMC-7721 cells with lipo2000-siBeclin 1 and LCP-II-siBeclin 1-FTY720, the apoptotic rates were $38 \%$ and $51 \%$, respectively. The co-delivery of siBeclin 1 and FTY720 with LCP-II NPs significantly increased apoptosis compared with that of the other groups (Figure 6A). Without FA, the effect of LCP-IIsiBeclin 1-FTY720 was significantly reduced to even less than that of free FTY720. The results showed that the suppression of autophagy by LCP-II-siBeclin 1-FTY720 NPs significantly increased cell death, which was associated with apoptosis induction.

Moreover, to further evaluate the levels of apoptotic proteins, such as cleaved caspase-3, cleaved caspase- 9 and cleaved PARP, in SMMC-7721 cells, we conducted Western blotting analyses of the protein extracts from the SMMC-7721 cells after treatment with different complexes. Consistent with the flow cytometry analysis results, treatment with free FTY720 and LCP-II-siRNA-FTY720 induced apoptosis. Additionally, compared with the levels in LCP-II-siRNA-FTY720- or free FTY720-treated cells, the addition of LCP-II-siBeclin 1-FTY720 to SMMC-7721 cells significantly increased the levels of cleaved caspase-3, caspase-9 and PARP, indicating that apoptosis had been induced (Figure 6B). In summary, these results indicated that co-delivery of siBeclin 1 and FTY720 with LCP-II NPs offers great potential for treatment of HCC.

\section{Tumor accumulation studies of LCP-II NPs}

To measure the effect of LCP-II NPs on tumor accumulation, we added Cy5-labeled siBeclin 1 to the LCP-II-siBeclin 1 and LCP-II-siBeclin 1-FTY720 NPs with or without FA to track tumor targeting with an in vivo imaging system. Tumorbearing male BALB/c nude mice were intravenously injected 


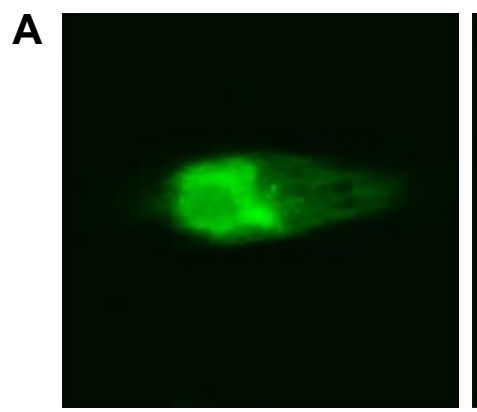

LCP-II-siRNA

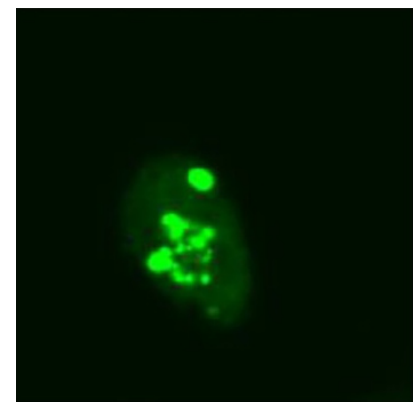

LCP-II-siRNA-FTY720

B

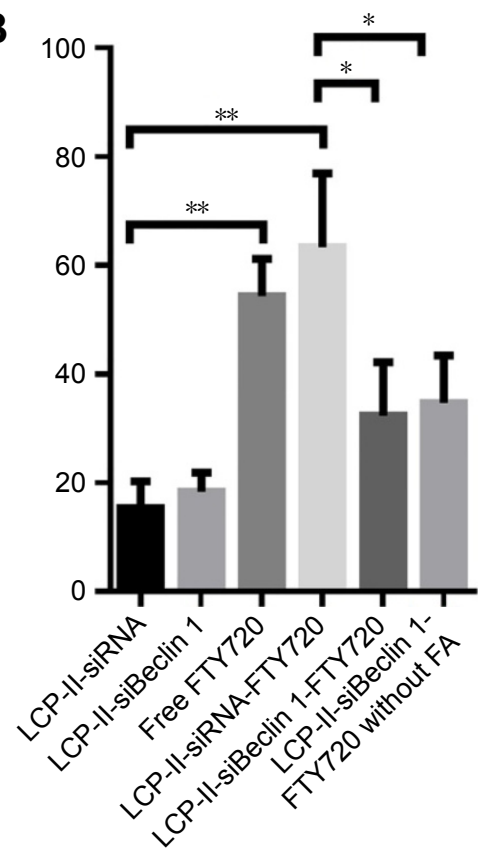

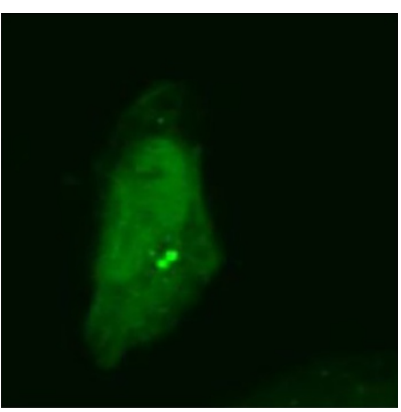

LCP-II-siBeclin 1

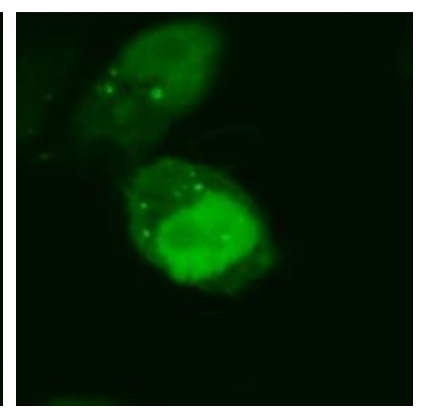

LCP-II-siBeclin 1-FTY720

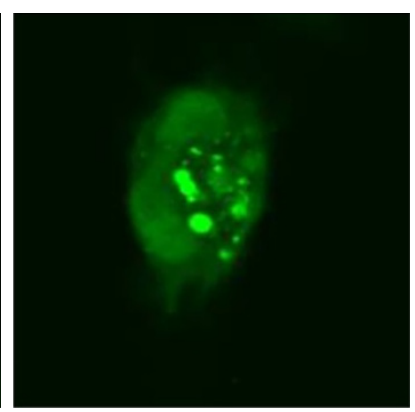

Free FTY720

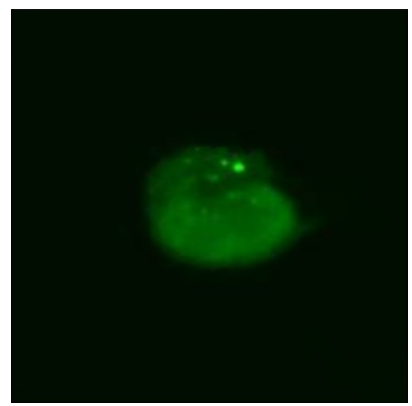

LCP-II-siBeclin 1-FTY720 without FA

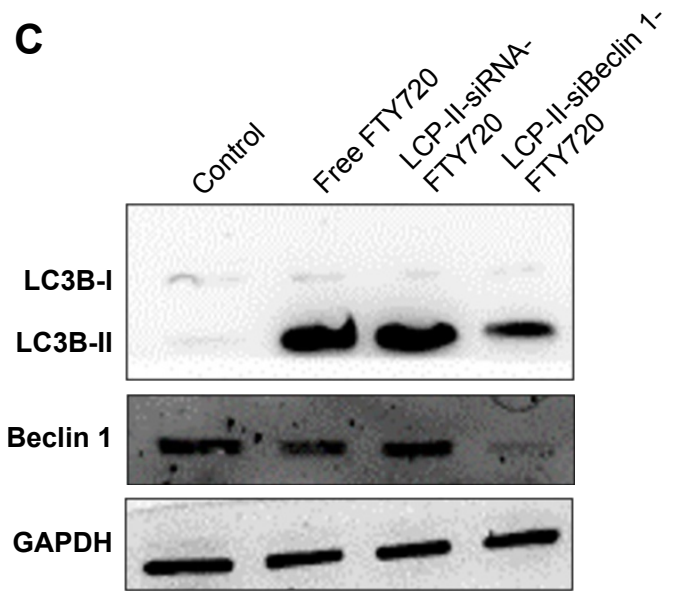

Figure 5 Formation of LC3 and GFP-LC3 puncta after treatment with LCP-II NPs.

Notes: (A) SMMC-772I cells were transfected with GFP-LC3 and treated with LCP-II-siRNA, LCP-II-siBeclin I or LCP-II-siBeclin I-FTY720 NPs with or without FA (total FTY720 $7.5 \mu \mathrm{M}$ ) or with $7.5 \mu \mathrm{M}$ FTY720 for 24 h; representative images of GFP-LC3 puncta formation are shown. (B) The percentage of cells presenting with typical GFP-LC3 puncta. (C) After treatment with PBS control, $7.5 \mu$ M FTY720, LCP-II-siRNA-FTY720 or LCP-II-siBeclin I-FTY720 (total FTY720 $7.5 \mu$ M) NPs for 24 h, the levels of LC3B-II and Beclin I were measured by Western blotting $(* P<0.05$, $* * P<0.01)$.

Abbreviations: FA, folic acid; FTY720, fingolimod; LCP-II NPs, lipid/calcium/phosphate type II nanoparticles; siBeclin I, Beclin I siRNA.

with PBS, LCP-II-siBeclin 1 or LCP-II-siBeclin 1-FTY720

NPs with or without FA at the given doses. After $12 \mathrm{~h}$, the Cy5 signal at the tumor site was much higher in mice treated with LCP-II-siBeclin 1 and LCP-II-siBeclin 1-FTY720 NPs with FA than in those administered LCP-II-siBeclin
1-FTY720 NPs without FA. Without FA, the LCP-II-siBeclin 1-FTY720 remained in other parts of the body and did not target the tumor (Figure 7A). We also investigated the effect of the co-delivery nanocarrier on biodistribution. After $4 \mathrm{~h}$, the Cy5 signal at the tumor site was much higher than in the 

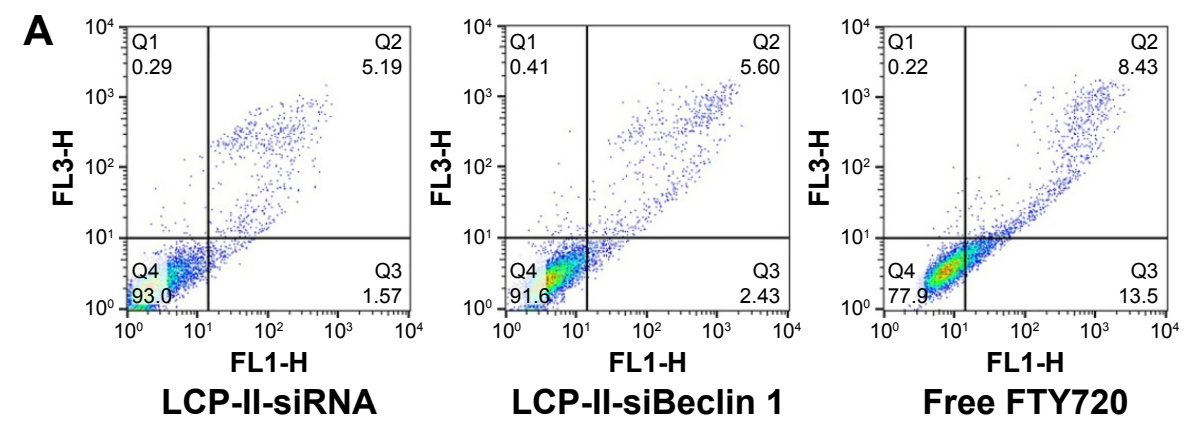

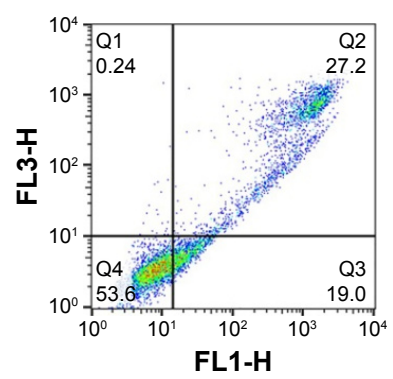

LCP-II-siRNAFTY720

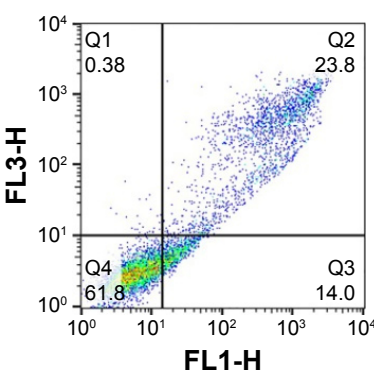

Lipo2000siBeclin 1 + FTY720

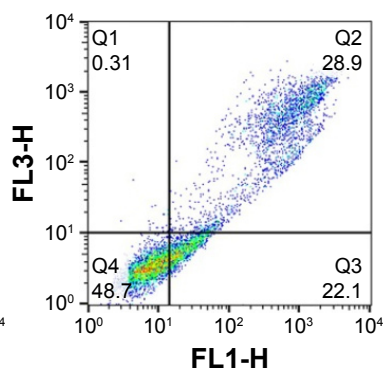

LCP-IIsiBeclin 1-FTY720

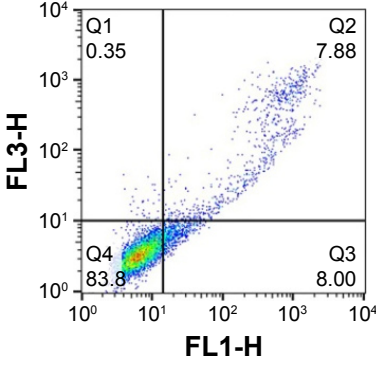

LCP-II-siBeclin 1FTY720 without FA

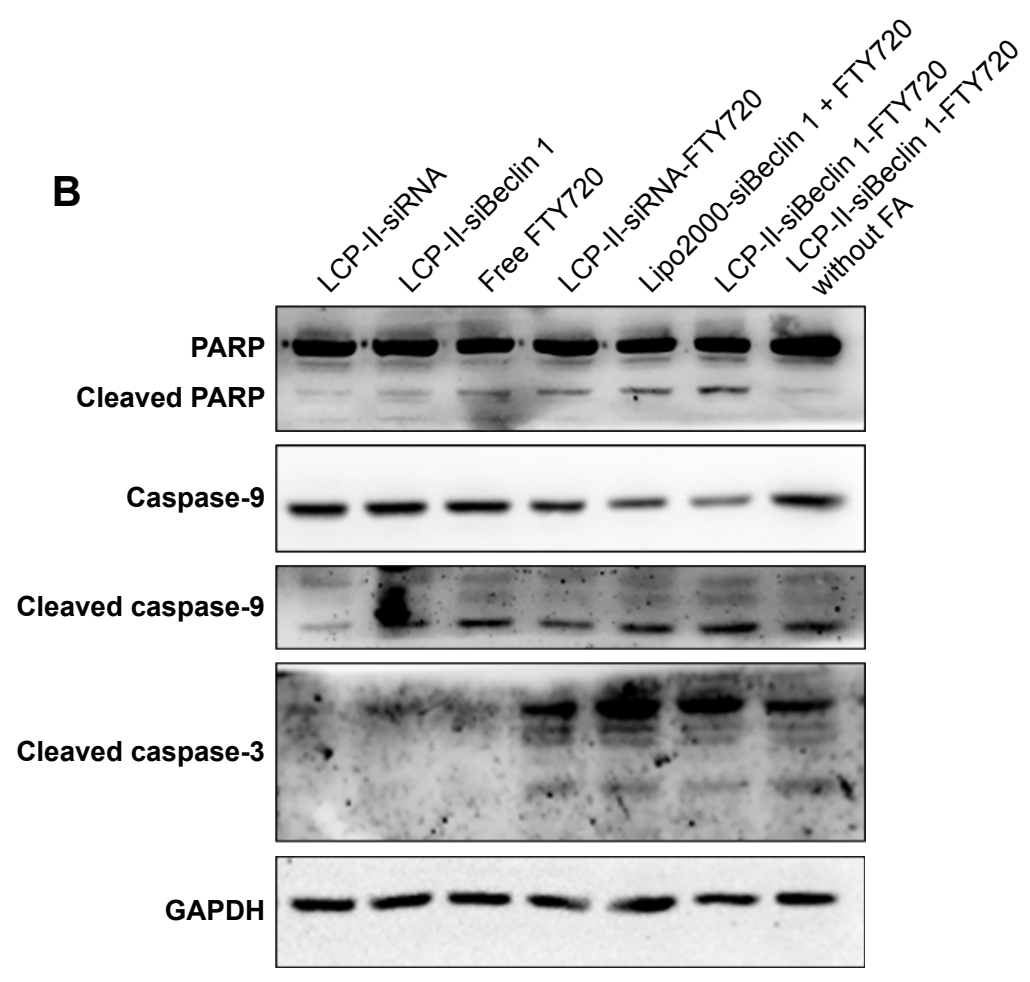

Figure 6 SMMC-772I cell apoptosis.

Notes: (A) After treatment with LCP-II-siRNA, LCP-II-siBeclin I, free FTY720, LCP-II-siRNA-FTY720, LCP-II-siBeclin I-FTY720 with or without FA or lipo2000-siBeclin I with FTY720 (7.5 $\mu \mathrm{M}$ total FTY720), the apoptosis rates were determined. (B) After treatment with LCP-II-siRNA, LCP-II-siBeclin I, free FTY720, LCP-II-siRNA-FTY720, LCP-II-siBeclin I-FTY720 with or without FA or lipo2000-siBeclin I and FTY720 (7.5 $\mu$ M total FTY720), the related protein levels were measured.

Abbreviations: FA, folic acid; FTY720, fingolimod; LCP-II NPs, lipid/calcium/phosphate type II nanoparticles; lipo2000, Lipofectamine 2000; siBeclin I, Beclin I siRNA.

liver and kidneys (Figure 7B), which confirmed that LCP-II was effectively delivered to the tumor site and preferred to accumulate in the tumor. The results indicated that targeting of FTY720 and siBeclin 1 to the tumor by co-delivery with LCP-II-siBeclin 1-FTY720 was feasible in vivo.

\section{In vivo antitumor efficacy of LCP-II NPs}

To determine whether co-delivery of siBeclin 1 and FTY720 with LCP-II NPs significantly enhanced the sensitivity of SMMC-7721 cells to FTY720 in vivo, we established a subcutaneous xenograft model in nude mice by intravenous 
A

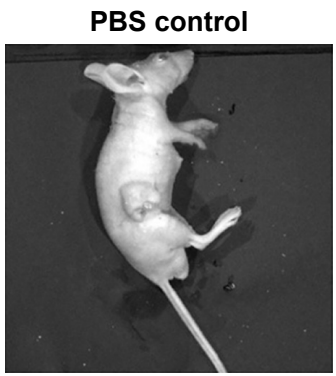

LCP-II-siBeclin 1FTY720
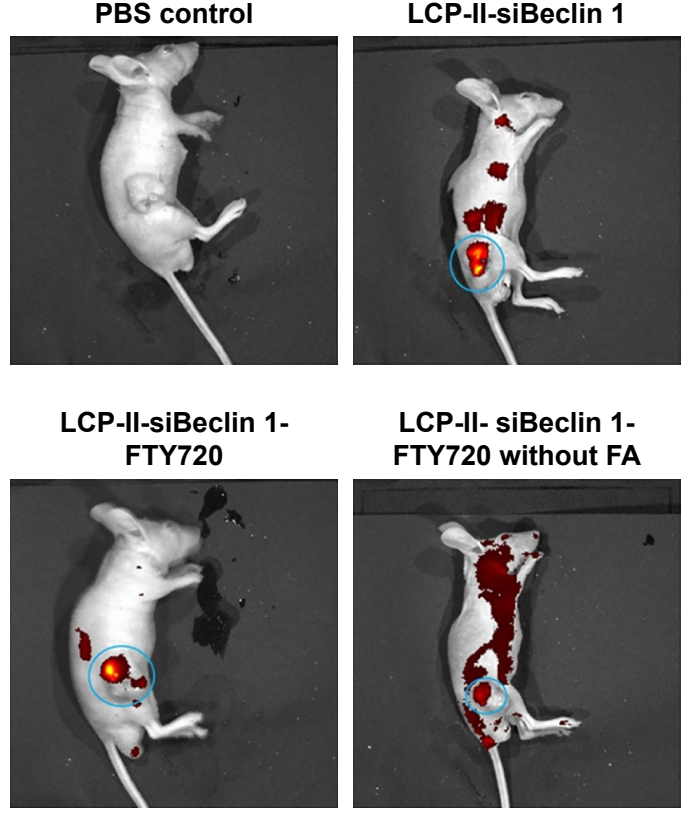
FTY720 without FA

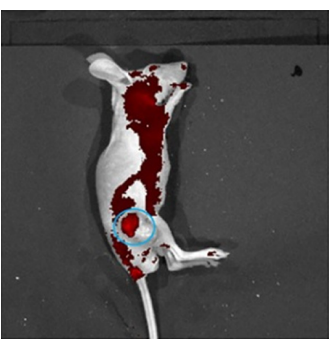

B

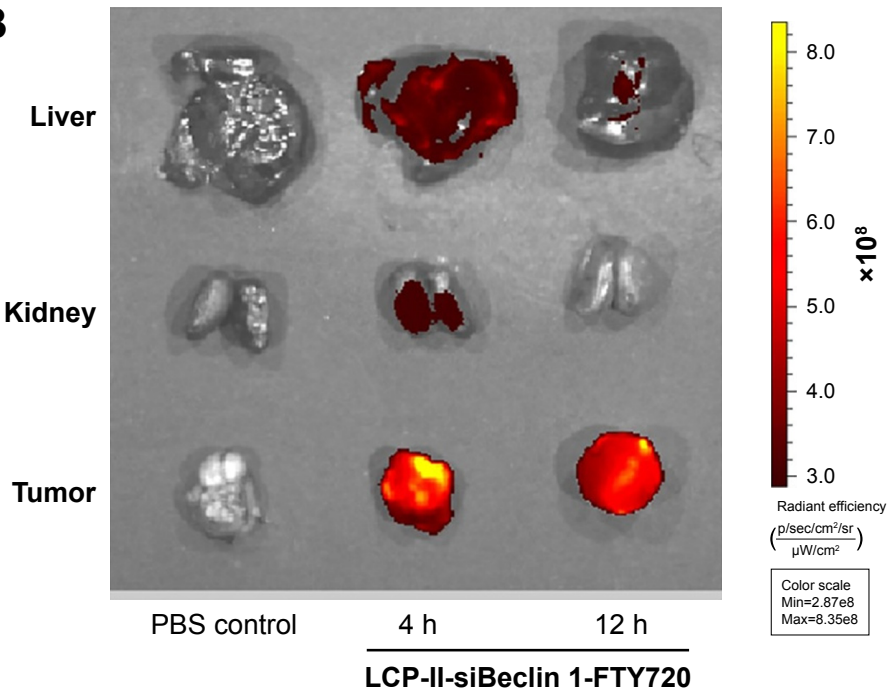

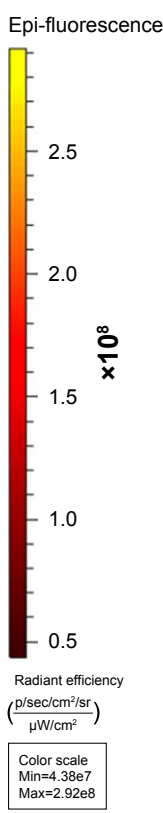
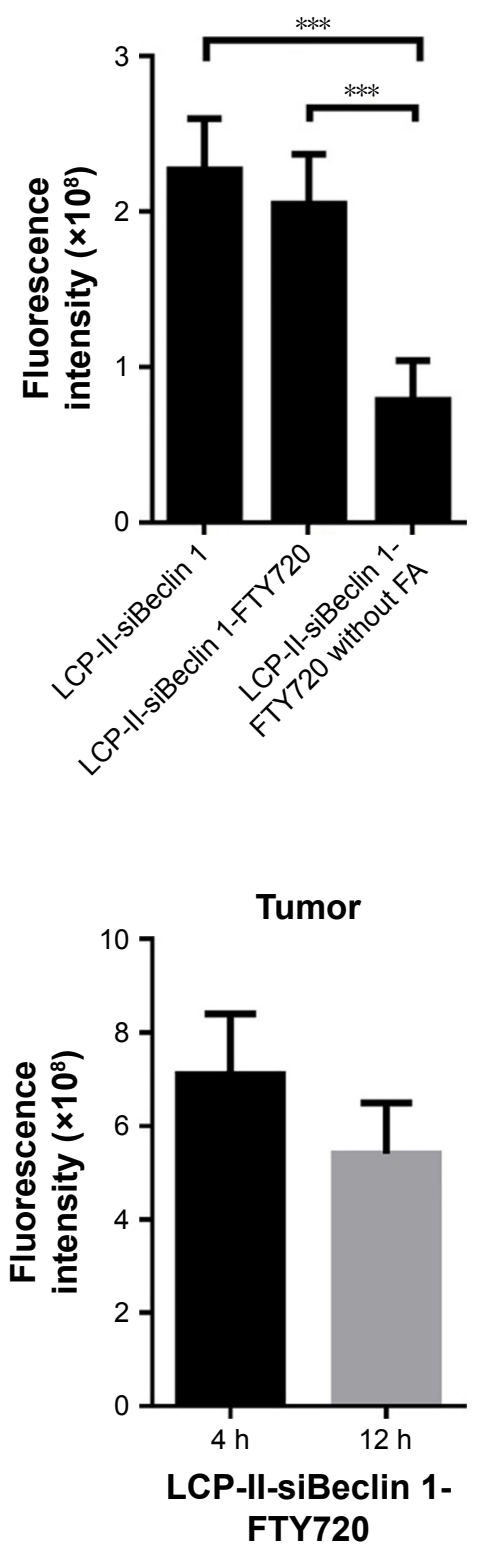

Figure 7 Evaluation of LCP-II NP tumor targeting.

Notes: (A) Whole body fluorescence imaging of mice after intravenous injection of the indicated formulations and quantitative analysis of fluorescence intensity at the tumor site (blue circle). (B) After 4 and I 2 h, the biodistribution of fluorescence-labeled siRNA delivered by LCP-II in the livers, kidneys and tumors of mice and quantitative analysis of fluorescence intensity at the tumor site. $(n=3 ; * * * P<0.00 I)$.

Abbreviations: FA, folic acid; FTY720, fingolimod; LCP-II NPs, lipid/calcium/phosphate type II nanoparticles; siBeclin I, Beclin I siRNA.

tail injection of different NP complexes to evaluate the antitumor efficacy. The results showed that LCP-II-siRNAFTY720 NP treatment caused a mild reduction in tumor volume and weight compared with the PBS-treated group. In addition, after treatment with LCP-II-siBeclin 1-FTY720 NPs, the tumors were significantly smaller than those in mice treated with LCP-II-siRNA-FTY720 NPs, indicating that the autophagy induced by FTY720 protected the cells from apoptosis. The volume and weight of tumors from nude mice treated with LCP-II-siBeclin 1-FTY720 NPs were four-fold less than those from nude mice treated with the PBS control. However, when we used LCP-II-siBeclin 1-FTY720 NPs without FA in mice, the antitumor efficacy was markedly reduced and even lower than that of LCPII-siRNA-FTY720 NPs with FA, which indicated that the FA ligand effectively identified and targeted SMMC-7721 cells and tumor xenografts in the nude mice (Figure 8A). In addition, there was no difference in the body weight of mice after the different treatments (Figure 8B), and no mice died in the experiment, which confirmed the low toxicity 

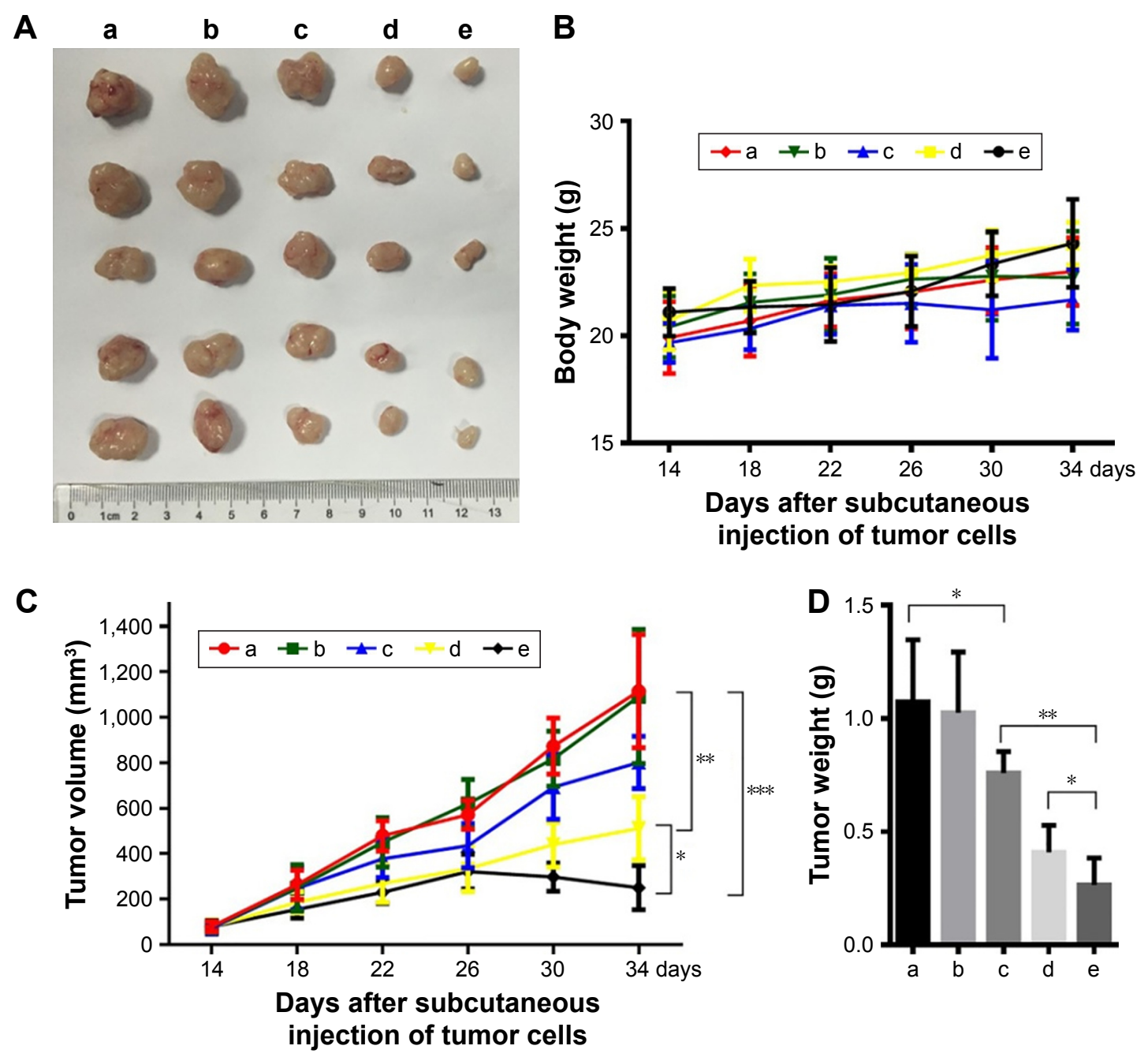

Figure 8 In vivo antitumor efficacy of treatment with different formulations.

Notes: (A) Tumors from mice treated with PBS control, LCP-II-siRNA negative control, LCP-II-siBeclin I-FTY720 NPs without FA, LCP-II-FTY720 NPs with FA and LCPII-siBeclin I-FTY720 NPs with FA (marked as a, b, c, d and e, respectively). (B) Body weights of mice during the injection period. (C) After tumors reached a volume of $50-100 \mathrm{~mm}^{3}$ ( 14 days after subcutaneous cell injection), treatment was initiated. (D) Tumor weights at the end of the experiment. ( $\mathrm{n}=5$; $* P<0.05$, $* * P<0.0 \mathrm{I}$, $* * * P<0.00 \mathrm{I}$ ). Abbreviations: FA, folic acid; FTY720, fingolimod; LCP-II NPs, lipid/calcium/phosphate type II nanoparticles; siBeclin I, Beclin I siRNA.

of LCP-II. These changes in tumor weight and volume are shown in Figure $8 \mathrm{C}$ and $\mathrm{D}$, confirming that co-delivering siRNA and drug with LCP-II NPs had a greater effect on inhibiting the growth of solid tumors than that induced by the FTY720-loaded LCP-II NPs.

However, more follow-up research is still needed to verify the toxic effects and mortality induced by the nanocarrier. In summary, these results indicated that co-delivery with nanocarriers and a targeting ligand is an effective new strategy for inhibiting tumor growth.

\section{Discussion}

FTY720 is a new immunomodulator that has been used with multiple sclerosis and multiple organ transplantation. ${ }^{10}$ Recently, FTY720 was found to inhibit the growth of various tumors, including HCC tumors..$^{40,41}$ Because of its nontoxicity, specific cytotoxicity toward tumors and high oral bioavailability, FTY720 has great potential for tumor therapy. However, after exploring the use of FTY720 in various cancers, studies have shown that this drug substantially induces autophagy in tumor cells, which protects them from damage and reduces apoptosis. ${ }^{10-12}$ Autophagy is a process of cell self-degradation that plays an important role in adapting to metabolic stress and maintaining genomic integrity and internal stability. ${ }^{42}$ When tumor cells experience prolonged starvation, hypoxia, radiotherapy or chemotherapeutics, they induce autophagy to reduce cellular damage and maintain cell stability. In some anticancer drug therapies, autophagy reduces apoptosis and promotes tumor resistance.

Recently, many studies have shown that inhibiting autophagy effectively enhances the cytotoxicity of FTY720. ${ }^{10,15}$ 
Other studies have found that suppressing Beclin 1 protein expression, which inhibits autophagy, ${ }^{43}$ with siRNA, substantially improves FTY720 sensitivity in cancer cells. In addition, an increasing number of studies have shown that the combined use of siRNA and anticancer drugs effectively reverses multidrug resistance and inhibits tumor growth. However, one of the major difficulties in combining drugs and siRNA therapy is targeting the tumor tissue. To solve the problem, studies have focused on using liposomal vehicles to co-deliver siRNA and anticancer drugs. ${ }^{28-30}$ For example, the co-delivery of doxorubicin and Bmil-siRNA with FA-modified cationic liposomes significantly enhanced tumor accumulation and improved the antitumor effect. ${ }^{44}$ Additionally, Yang et al designed tumor-targeting cationic liposomes modified with dual peptides for co-delivery of siRNA and doxorubicin to gliomas. ${ }^{45}$ In summary, combining siRNA and anticancer drugs into the same vector has unique advantages.

In our study, we tested the ability of a novel siRNA delivery vector, LCP-II NPs, to co-deliver Beclin 1 siRNA and FTY720, and we demonstrated the protective role of FTY720-induced autophagy in reducing apoptosis. The results revealed that free FTY720 and LCP-II-siRNAFTY720 NPs induced cell death and that the LCP-II-siRNAFTY720 NPs were more effective than the free drug. As previously shown, free FTY720 and LCP-II-siRNA-FTY720 NPs also induced cell autophagy. After introduction of Beclin 1 siRNA, the Beclin 1 protein expression was effectively reduced, leading to autophagy inhibition. A series of experiments, such as confocal microscopy of GFP-LC3transfected SMMC-7721 cells and Western blot analysis of the LC3-II to LC3-I ratio, confirmed the role of siBeclin 1 in LCP-II-siBeclin 1-FTY720 NPs. The results showed that after treatment with LCP-II-siBeclin 1-FTY720 NPs, the proportion of SMMC-7721 cells forming LC3 puncta and the ratio of LC3-II to LC3-I were significantly decreased, indicating that autophagy was suppressed by the co-delivery NPs. In addition, after treatment with siBeclin 1 and FTY720 through the co-delivery LCP-II NPs, cell viability was significantly decreased. Furthermore, the ratio of AV-positive and PI-positive cells increased to $50 \%$ after treatment with the LCP-II-siBeclin 1-FTY720 NPs for $24 \mathrm{~h}$, and the expression levels of cleaved caspase-3, caspase-9 and PARP were also increased compared with the levels in cells treated with free FTY720 and LCP-II-siRNA-FTY720 NPs. Consistent with the in vitro experiments, co-delivery of siBeclin 1 and FTY720 in vivo exhibited the most powerful antitumor efficacy. Tumor weight and volume were significantly smaller in the LCP-II-siBeclin 1-FTY720 NP-treated group than in the other groups. However, without FA targeting, the cytotoxicity of LCP-II-siBeclin 1-FTY720 NPs was substantially decreased, indicating that this ligand was essential for targeted delivery to the tumor site. Consequently, blocking autophagy enhanced the cytotoxicity of FTY720. Autophagy is most likely an important factor in HCC resistance to FTY720. Combined treatment with siBeclin 1 and FTY720 using LCP-II NPs may effectively promote the anticancer efficacy of FTY720 and has great potential for HCC treatment.

\section{Conclusion}

LCP-II NPs effectively encapsulate siRNA and anticancer drugs, resulting in particles with a suitable average size that are internalized by SMMC-7721 cells. Treatment with these NPs silences Beclin 1 expression, blocking the autophagy induced by FTY720. Compared with free FTY720 and LCP-II-siRNA-FTY720 NPs alone, combined delivery of siBeclin 1 and FTY720 more efficiently inhibited HCC cell progression by suppressing protective autophagy and increasing apoptosis. This is the first time we have used LCP-II NPs as a co-delivery system for a small molecule and siRNA, and we found that this system has favorable synergistic effects for cancer treatment. Combining gene therapy and anticancer drugs in a nanocarrier is a popular focus of nonsurgical tumor treatment.

\section{Acknowledgments}

This research was supported by the National Natural Science Foundation of China (nos 81572393, 81602093, $81773255,81472820,81602054)$, the Key Project supported by the Medical Science and Technology Development Foundation, Nanjing Municipality Health Bureau (no ZKX15020), the Natural Science Foundation of Jiangsu Province (nos BK20141324 and BK20160118), the National Basic Research Program of China (973 Program, 2013CB834100) and Jiangsu special program for clinical medical science and technology (BL2014054). We thank the Translational Medicine Core facilities of the Medical School of Nanjing University for instrument support.

\section{Authors contributions}

All authors contributed toward data analysis, drafting and revising the paper and agree to be accountable for all aspects of the work. JYW and ZXW contributed equally to this work. 


\section{Disclosure}

The authors report no competing interests in this work.

\section{References}

1. Torre LA, Bray F, Siegel RL, Ferlay J, Lortet-Tieulent J, Jemal A. Global cancer statistics, 2012. CA Cancer J Clin. 2015;65(2):87-108.

2. El-Serag HB, Rudolph KL. Hepatocellular carcinoma: epidemiology and molecular carcinogenesis. Gastroenterology. 2007;132(7):2557-2576.

3. Yao FY, Ferrell L, Bass NM, et al. Liver transplantation for hepatocellular carcinoma: expansion of the tumor size limits does not adversely impact survival. Hepatology. 2001;33(6):1394-1403.

4. Bruix J, Gores GJ, Mazzaferro V. Hepatocellular carcinoma: clinical frontiers and perspectives. Gut. 2014;63(5):844-855.

5. de Lope CR, Tremosini S, Forner A, Reig M, Bruix J. Management of HCC. J Hepatol. 2012;56(Suppl 1):S75-S87.

6. Kumar V, Bhatt PC, Rahman M, et al. Fabrication, optimization, and characterization of umbelliferone beta-D-galactopyranoside-loaded PLGA nanoparticles in treatment of hepatocellular carcinoma: in vitro and in vivo studies. Int $J$ Nanomedicine. 2017;12:6747-6758.

7. Pyne NJ, Pyne S. Sphingosine 1-phosphate and cancer. Nat Rev Cancer. 2010;10(7):489-503

8. Azuma H, Takahara S, Ichimaru N, et al. Marked prevention of tumor growth and metastasis by a novel immunosuppressive agent, FTY720, in mouse breast cancer models. Cancer Res. 2002;62(5):1410-1419.

9. Perrotti D, Neviani P. Protein phosphatase 2A: a target for anticancer therapy. Lancet Oncol. 2013;14(6):e229-e238.

10. Zhang N, Qi Y, Wadham C, Wang L, Warren A, Di W, Xia P. FTY720 induces necrotic cell death and autophagy in ovarian cancer cells: a protective role of autophagy. Autophagy. 2010;6(8):1157-1167.

11. Li J, Wang SW, Zhang DS, et al. FTY720-induced enhancement of autophagy protects cells from FTY720 cytotoxicity in colorectal cancer. Oncol Rep. 2016;35(5):2833-2842.

12. Marvaso G, Barone A, Amodio N, et al. Sphingosine analog fingolimod (FTY720) increases radiation sensitivity of human breast cancer cells in vitro. Cancer Biol Ther. 2014;15(6):797-805.

13. Levine B, Kroemer G. Autophagy in the pathogenesis of disease. Cell. 2008;132(1):27-42.

14. Wang GD, Tan YZ, Wang HJ, Zhou P. Autophagy promotes degradation of polyethyleneimine-alginate nanoparticles in endothelial progenitor cells. Int J Nanomedicine. 2017;12:6661-6675.

15. Maiuri MC, Zalckvar E, Kimchi A, Kroemer G. Self-eating and selfkilling: crosstalk between autophagy and apoptosis. Nat Rev Mol Cell Biol. 2007;8(9):741-752.

16. Ou L, Lin S, Song B, Liu J, Lai R, Shao L. The mechanisms of graphenebased materials-induced programmed cell death: a review of apoptosis, autophagy, and programmed necrosis. Int J Nanomedicine. 2017;12: 6633-6646.

17. Wallington-Beddoe CT, Hewson J, Bradstock KF, Bendall LJ. FTY720 produces caspase-independent cell death of acute lymphoblastic leukemia cells. Autophagy. 2011;7:707-715.

18. Patmanathan SN, Yap LF, Murray PG, Paterson IC. The antineoplastic properties of FTY720: evidence for the repurposing of fingolimod. J Cell Mol Med. 2015;19:2329-2340.

19. Tay KH, Liu X, Chi M, et al. Involvement of vacuolar $\mathrm{H}(+)$-ATPase in killing of human melanoma cells by the sphingosine kinase analogue FTY720. Pigment Cell Melanoma Res. 2015;28:171-183.

20. Zuckerman JE, Davis ME. Clinical experiences with systemically administered siRNA-based therapeutics in cancer. Nat Rev Drug Discov. 2015;14(12):843-856.

21. Chen Y, Wang X, Liu T, et al. Highly effective antiangiogenesis via magnetic mesoporous silica-based siRNA vehicle targeting the VEGF gene for orthotopic ovarian cancer therapy. Int J Nanomedicine. 2015; 10:2579-2594.

22. Meng H, Liong M, Xia T, et al. Engineered design of mesoporous silica nanoparticles to deliver doxorubicin and P-glycoprotein siRNA to overcome drug resistance in a cancer cell line. ACS Nano. 2010;4(8):4539-4550.
23. Wang Y, Gao S, Ye WH, Yoon HS, Yang YY. Co-delivery of drugs and DNA from cationic core-shell nanoparticles self-assembled from a biodegradable copolymer. Nat Mater. 2006;5(10):791-796.

24. Cheng D, Cao N, Chen J, Yu X, Shuai X. Multifunctional nanocarrier mediated co-delivery of doxorubicin and siRNA for synergistic enhancement of glioma apoptosis in rat. Biomaterials. 2012;33(4): $1170-1179$.

25. Biswas S, Deshpande PP, Navarro G, Dodwadkar NS, Torchilin VP. Lipid modified triblock PAMAM-based nanocarriers for siRNA drug co-delivery. Biomaterials. 2013;34(4):1289-1301.

26. Muthu MS, Leong DT, Mei L, Feng SS. Nanotheranostics - application and further development of nanomedicine strategies for advanced theranostics. Theranostics. 2014;4(6):660-677.

27. Gandhi NS, Tekade RK, Chougule MB. Nanocarrier mediated delivery of siRNA/miRNA in combination with chemotherapeutic agents for cancer therapy: current progress and advances. $J$ Control Release. 2014;194:238-256.

28. Saenz del Burgo L, Pedraz JL, Orive G. Advanced nanovehicles for cancer management. Drug Discov Today. 2014;19(10):1659-1670.

29. Zheng Y, Su C, Zhao L, Shi Y. Chitosan nanoparticle-mediated co-delivery of shAtg-5 and gefitinib synergistically promoted the efficacy of chemotherapeutics through the modulation of autophagy. J Nanobiotechnology. 2017;15(1):28.

30. Babu A, Munshi A, Ramesh R. Combinatorial therapeutic approaches with RNAi and anticancer drugs using nanodrug delivery systems. Drug Dev Ind Pharm. 2017;43(9):1391-1401.

31. Kang SH, Cho HJ, Shim G, et al. Cationic liposomal co-delivery of small interfering RNA and a MEK inhibitor for enhanced anticancer efficacy. Pharm Res. 2011;28(12):3069-3078.

32. Ding L, Sun R, Zhang X. Rap2b siRNA significantly enhances the anticancer therapeutic efficacy of adriamycin in a gold nanoshell-based drug/ gene co-delivery system. Oncotarget. 2017;8(13):21200-21211.

33. Kato T, Natsume A, Toda H, et al. Efficient delivery of liposomemediated MGMT-siRNA reinforces the cytotoxity of temozolomide in GBM-initiating cells. Gene Ther. 2010;17(11):1363-1371.

34. Yu M, Han S, Kou Z, et al. Lipid nanoparticle-based co-delivery of epirubicin and BCL-2 siRNA for enhanced intracellular drug release and reversing multidrug resistance. Artif Cells Nanomed Biotechnol. 2018:46(2):323-332.

35. Li J, Yang Y, Huang L. Calcium phosphate nanoparticles with an asymmetric lipid bilayer coating for siRNA delivery to the tumor. J Control Release. 2012;158(1):108-114.

36. Lu B, Gao Q, Liu R, et al. Effect of a new drug releasing system on microencapsulated islet transplantation. Int J Clin Exp Pathol. 2015; 8(10):12390-12399.

37. Erdogar N, Esendagli G, Nielsen TT, et al. Therapeutic efficacy of folate receptor-targeted amphiphilic cyclodextrin nanoparticles as a novel vehicle for paclitaxel delivery in breast cancer. J Drug Target. 2018; 26(1):66-74.

38. Thapa RK, Choi JY, Poudel BK, Choi HG, Yong CS, Kim JO. Receptortargeted, drug-loaded, functionalized graphene oxides for chemotherapy and photothermal therapy. Int J Nanomedicine. 2016;11:2799-2813.

39. Ding N, Lu Y, Lee RJ, et al. Folate receptor-targeted fluorescent paramagnetic bimodal liposomes for tumor imaging. Int J Nanomedicine. 2011;6:2513-2520.

40. Ahmed D, de Verdier PJ, Ryk C, Lunqe O, Stal P, Flygare J. FTY720 (Fingolimod) sensitizes hepatocellular carcinoma cells to sorafenibmediated cytotoxicity. Pharmacol Res Perspect. 2015;3(5):e00171.

41. Hung JH, Lu YS, Wang YC, et al. FTY720 induces apoptosis in hepatocellular carcinoma cells through activation of protein kinase $\mathrm{C}$ delta signaling. Cancer Res. 2008;68(4):1204-1212.

42. Wang J, Yu Y, Lu K, et al. Silica nanoparticles induce autophagy dysfunction via lysosomal impairment and inhibition of autophagosome degradation in hepatocytes. Int J Nanomedicine. 2017;12:809-825.

43. Liang $\mathrm{C}$, Feng $\mathrm{P}, \mathrm{Ku} \mathrm{B}$, et al. Autophagic and tumour suppressor activity of a novel Beclin1-binding protein UVRAG. Nat Cell Biol. 2006;8(7): 688-699. 
44. Yang T, Li B, Qi S, et al. Co-delivery of doxorubicin and Bmil siRNA by folate receptor targeted liposomes exhibits enhanced anti-tumor effects in vitro and in vivo. Theranostics. 2014;4(11):1096-1111.
45. Yang ZZ, Li JQ, Wang ZZ, Dong DW, Qi XR. Tumor-targeting dual peptides-modified cationic liposomes for delivery of siRNA and docetaxel to gliomas. Biomaterials. 2014;35(19):5226-5239.

\section{Publish your work in this journal}

The International Journal of Nanomedicine is an international, peerreviewed journal focusing on the application of nanotechnology in diagnostics, therapeutics, and drug delivery systems throughout the biomedical field. This journal is indexed on PubMed Central, MedLine, CAS, SciSearch $®$, Current Contents ${ }^{\circledR} /$ Clinical Medicine,
Journal Citation Reports/Science Edition, EMBase, Scopus and the Elsevier Bibliographic databases. The manuscript management system is completely online and includes a very quick and fair peer-review system, which is all easy to use. Visit http://www.dovepress.com/ testimonials.php to read real quotes from published authors. 